\title{
Thermal inertia at the MSL and InSight mission sites on Mars
}

\author{
D. Singh ${ }^{1 *}$, and S. Uttam ${ }^{2}$ \\ ${ }^{1}$ Centre of Studies in Resources Engineering, Indian Institute of Technology Bombay, Mumbai, India; \\ 2Physical Research Laboratory, Ahmedabad, India
}

Key Points:

- Surface thermal inertia determination using MSL and InSight ground measurements.

- Dust storms have significant impact on surface's thermal inertia and albedo.

- Variation in bulk properties at two InSight sites; surface composition changes.

Citation: Singh, D., and Uttam, S. (2022). Thermal inertia at the MSL and InSight mission sites on Mars. Earth Planet. Phys., 6(1), 18-27. http://doi.org/10.26464/epp2022004

\begin{abstract}
For planetary surface materials, thermal inertia is the critical property that governs the surface's daily thermal response and controls diurnal and seasonal surface temperature variations. Here we use the ground measurements made by the MSL Curiosity rover and the InSight lander to determine the thermal inertia of two sites on Mars. This study compares the variation of thermal inertia during and after the Large Dust Storm (LDS) of Martian Year (MY) 34. To determine surface thermal inertia, we derive a simple approximation (using energy balance), which utilizes surface albedo, surface energy flux, and diurnal change in the surface temperature. The average thermal inertia in MY34 is about 39.2\%, 3.7\%, and 3.4\% higher than MY35 average thermal inertia for the MSL, InSight (FOV1), and InSight (FOV2), respectively. Notably, the thermal inertia at the InSight (FOV1) is consistently lower by about $20 \mathrm{~J} \cdot \mathrm{m}^{-2} \cdot \mathrm{s}^{-1 / 2} \cdot \mathrm{K}^{-1}$ than the InSight (FOV2) site for all scenarios, indicating variation in the region's surface composition. The best-fit surface albedo in MY34 (determined using the KRC model) are about 0.08, 0.05, and 0.03 higher than MY35 surface albedo for the MSL, InSight (FOV1), and InSight (FOV2), respectively. An increase in both surface albedo and thermal inertia during the LDS indicates that the underlying surface is both more thermally resistant and more reflective than the overlying loose dust.
\end{abstract}

Keywords: Mars; Mars surface; thermal inertia; dust storm; Mars climate

\section{Introduction}

The surface thermal inertia is a crucial property for understanding the subsurface heat flow of Mars and determining the forcing function for subsurface temperatures. The near-surface temperature is highly influenced by surface thermal inertia and albedo properties, as well as atmospheric dust opacity (e.g., Spiga and Forget, 2009; Martínez et al., 2014). Apart from impacting nearsurface temperatures, heat flow variations can also affect the planet's internal geodynamics, ice/snow distribution, and processes such as subsurface melting on Mars (Parro et al., 2017). The surface thermal inertia determines the temperature response of the surface to incident energy flux. Compared to Earth, Mars' surface thermal inertia plays a more significant role in its climate because the atmospheric density is much lower and the surface also lacks oceans, which act as heat reservoirs.

Thermal inertia is the capacity of a material to sustain or transmit heat. For planetary surface materials, thermal inertia is the critical property that governs the surface's daily thermal response and controls diurnal and seasonal surface temperature variations (e.g.,

Correspondence to: D. Singh, sdeepak@umich.edu

Received 14 JUN 2021; Accepted 06 OCT 2021.

Accepted article online 24 DEC 2021.

(C) 2022 by Earth and Planetary Physics.
Putzig et al., 2005; Vu et al., 2019). The thermal inertia of the planetary surface is typically dependent on the physical properties of near-surface geologic materials.

The thermal inertia $(I)$ of a surface $\left(\mathrm{J} \cdot \mathrm{m}^{-2} \cdot \mathrm{s}^{-1 / 2} \cdot \mathrm{K}^{-1}\right)$ is defined as:

$$
I=\sqrt{k \rho c},
$$

where, $k$ is the bulk thermal conductivity $\left(\mathrm{W} \cdot \mathrm{m}^{-1} \cdot \mathrm{K}^{-1}\right), \rho$ is the bulk density $\left(\mathrm{kg} \cdot \mathrm{m}^{-3}\right)$, and $\mathrm{c}$ is the bulk specific heat capacity (J. $\mathrm{kg}^{-1} \cdot \mathrm{K}^{-1}$ ) of the material.

Generally, thermal inertia (or similar surface properties) are determined via orbiter data for a distant object like Mars with the advantage of covering the entire planet in a relatively short period of time. However, this approach also limits our knowledge of local surface physical properties because of the resolution limitation of orbiter data. Bulk surface properties like thermal inertia are crucial for understanding local atmospheric phenomena. The surface thermal inertia varies significantly over small distances depending on local topography and composition (Christensen et al., 2001; Martínez et al., 2014; Mellon et al., 2000; Piqueux et al., 2019). Previous studies have primarily used complicated and lengthy numerical models for solving radiative transfer and other related equations to determine thermal inertia, tuning the model by varying surface parameters like albedo, thermal inertia, and 
dust opacity to match in-situ temperature measurements.

In this study, we utilize a simplistic energy balance approach (details in Section 3) to determine the thermal inertia at two Mars mission sites. This approach does not require knowledge of the entire radiative transfer solution through the Martian atmosphere, solving complicated numerical models and the surface's bulk properties. Here we analyze the data that includes the Large Dust Storm (LDS) of MY34 (Guzewich et al., 2019; Montabone et al., 2020; Streeter et al., 2020; Viúdez-Moreiras et al., 2020) in comparison to the non-dusty period of MY35 to infer the impact of dust on the surface thermal inertia. In the subsequent sections, we will detail the missions and data sources, methodology, the variation of thermal inertia from dusty to non-dust period, comparison of observed and model-derived diurnal temperatures, and best-fit albedo variations. Our approach independently determines the thermal inertia directly from temperature measurements and does not include any tuning for the surface thermal inertia determination.

\section{Mission and Data Description}

We use data from the Rover Environmental Monitoring Station (REMS) instrument onboard the Mars Science Laboratory (MSL) rover Curiosity (Gómez-Elvira et al., 2012), and the Heat Flow and Physical Properties Package/Radiometer (HP3/RAD) data onboard the InSight Lander (Spohn et al., 2018). MSL landed with the Curiosity rover in Gale crater $\left(5.4^{\circ} \mathrm{S}, 137.8^{\circ} \mathrm{E}\right)$ on $6^{\text {th }}$ August 2012 at solar longitude (Ls) $\sim 151^{\circ}$ (during the late Martian southern hemisphere winter) (Haberle et al., 2014; Savijärvi et al., 2015; Wall, 2012). InSight landed successfully in the western Elysium Planitia region $\left(4.502^{\circ} \mathrm{N}, 135.623^{\circ} \mathrm{E}\right)$ on $26^{\text {th }}$ November 2018 at Ls $\sim 295^{\circ}$ (during the late Martian southern hemisphere summer) (Banerdt et al., 2020; Banfield et al., 2020; Golombek et al., 2020). Figure 1 shows the MSL Curiosity rover and the InSight lander approximate locations overlaid on the Mars Orbiter Laser Altimeter (MOLA) topographic map (Smith et al., 1999; Zuber et al., 1992). Figure 1 also depicts some key past and present Mars' mission locations. Viking Lander 1 and Viking Lander 2 are indicated as VL1 and VL2, respectively.
The REMS instrument onboard MSL measures air temperature, ground temperature, atmospheric pressure, wind speed and direction, atmospheric relative humidity, and UV radiation fluxes (Gómez-Elvira et al., 2012). The REMS instrument collects radiance in three bandpasses for brightness temperature measurements, however, only the 8-14 $\mu \mathrm{m}$ bandpass achieves a resolution of $2 \mathrm{~K}$ (or better) and an accuracy of $10 \mathrm{~K}$ (or better) from 150 to $300 \mathrm{~K}$ (Gómez-Elvira et al., 2012; Vasavada et al., 2017).

The purpose of the $\mathrm{HP}^{3}$ instrument is to determine the geothermal heat flow at the landing site. The RAD instrument (Kopp et al., 2016) augments the HP3 instrument by measuring the surface brightness temperature that determines the subsurface temperatures' forcing function. The RAD instrument also has three different bandpasses: the primary broad bandpass from 8-14 $\mu \mathrm{m}$, and the other two filters from 8-10 $\mu \mathrm{m} \& 15-19 \mu \mathrm{m}$. The primary broad bandpass is used for retrieving surface temperatures and the other two filters to resolve any inhomogeneous temperature distribution (Kopp et al., 2016; Mueller et al., 2020; Spohn et al., 2018). The effective surface emissivity in the $8-14 \mu \mathrm{m}$ band is likely to range from 0.95 to 0.99 (Morgan et al., 2018). The RAD instrument has a random measurement uncertainty of $4 \mathrm{~K}$ at $150 \mathrm{~K}$ brightness temperature, which is less significant than the possible error from the unknown emissivity (Kopp et al., 2016; Spohn et al., 2018). The RAD instrument measures brightness temperatures at two different fields of view (FOVs), one close to the lander (FOV1) and another farther from the lander (FOV2) (Mueller et al., 2020).

In this study, we utilize MSL brightness temperature data from $L s=294^{\circ}$ to $L s=360^{\circ}$ for MY34 and from $L s=0^{\circ}$ to $L s=103^{\circ}$ for MY35 (Gómez-Elvira, 2013a, 2013b). For InSight, we utilize derived surface temperatures from $L s=304^{\circ}$ to $L s=360^{\circ}$ for $M Y 34$ and from $L s=0^{\circ}$ to $L s=127^{\circ}$ for MY35. These datasets cover both the dusty conditions in MY34 and non-dusty conditions in MY35. Also, analyzing the same time duration from both the missions enables us to carry out a fair comparison between both missions' thermal properties. We excluded RAD data (from InSight mission) with any fraction of lander shadow on either FOV to remove the effect of shadow on thermal inertia retrievals (Mueller et al., 2020;

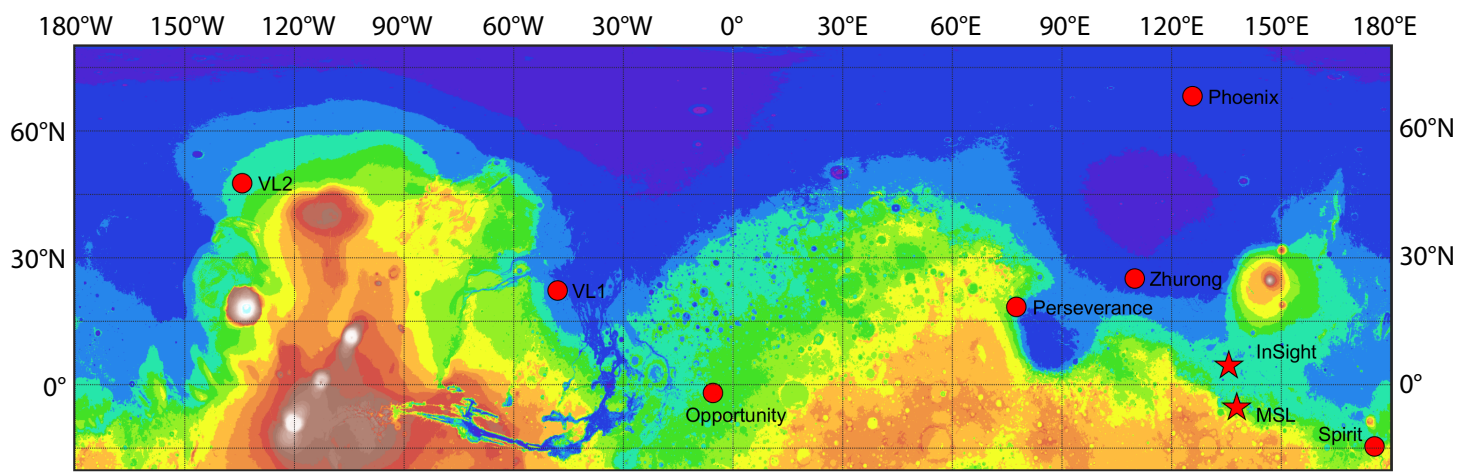

Elevation $(\mathrm{km})$

\begin{tabular}{|cccccccc|}
\hline & -4 & 1 & & & 1 & 1 & 1 \\
\hline-8 & -4 & 0 & 4 & 8 & 12 & 16 & 20
\end{tabular}

Figure 1. Locations of the MSL Curiosity rover and the InSight lander (both marked as stars) along with some other key landers/rovers past and present Mars' missions (marked as circles) on the MOLA topographic map. VL1 and VL2 indicate Viking landers 1 and 2, respectively. 
Piqueux et al., 2019; Spohn et al., 2018).

\section{Methodology}

The depth at which the thermal wave amplitude is attenuated by a factor of 1/e is known as the diurnal skin depth. The thermal diurnal skin depth is a measure of how far a diurnal surface temperature cycle penetrates the surface. The diurnal skin depth $(\delta)$ of a surface is defined as e.g., (Jakosky, 1986; Putzig and Mellon, 2007; Hamm et al., 2018; Vu et al., 2019):

$$
\delta=\sqrt{\frac{k P}{\rho c \pi}}=\frac{I}{\rho c} \sqrt{\frac{P}{\pi}}
$$

where $P$ is the period of the insolation cycle (88640 sec for Mars).

The total energy absorbed by the surface during the day is given as (Martínez et al., 2014; Tian et al., 2015; Wang et al., 2010):

$$
Q_{\mathrm{abs}}=\rho c \delta \Delta T=I \Delta T \sqrt{\frac{P}{\pi^{\prime}}}
$$

where $\Delta T$ is the diurnal amplitude of surface temperature (daynight temperature difference).

For a surface with albedo $a$, the total absorbed solar flux at the surface (using energy balance) would be given as:

$$
F_{\text {abs }}=(1-a) F_{0}
$$

where $F_{0}$ is the daily mean incident solar energy flux $\left(\mathrm{W} \cdot \mathrm{m}^{-2}\right)$. The total energy absorbed by the surface during a sol can also be given as:

$$
\begin{gathered}
Q_{\mathrm{abs}}=F_{\mathrm{abs}} P, \\
Q_{\mathrm{abs}}=(1-a) F_{0} P .
\end{gathered}
$$

Using Equations (3) and (6), the thermal inertia of a surface can be derived as,

$$
I=\frac{(1-a) F_{0} \sqrt{P \pi}}{\Delta T}
$$

Equation (7) provides a simple approximation of the surface thermal inertia in terms of surface albedo, incident solar energy flux, and diurnal change in the surface temperature. Therefore, thermal inertia can be determined if simultaneous surface energy flux and surface temperature measurements are available.

The KRC is a 1-D numerical thermal model that has been extensively used to estimate planetary surface temperatures (Kieffer, 2013; Vasavada et al., 2017). The KRC model uses thermal inertia, albedo, elevation, slope, and zonal climate to compute both diurnal and annual average surface temperatures (Kieffer, 2013). KRC solves the heat diffusion equation and accounts for first-order effects of the atmosphere. For this study, we use thermal inertia (previously known) values of $403 \mathrm{~J} \cdot \mathrm{m}^{-2} \cdot \mathrm{s}^{-1 / 2} \cdot \mathrm{K}^{-1}$ and $228 \mathrm{~J} \cdot \mathrm{m}^{-2} \cdot \mathrm{s}^{-1 / 2} \cdot \mathrm{K}^{-1}$ for MSL and InSight sites, respectively (e.g., Christensen and Moore, 1992; Jakosky et al., 2000; Mellon et al., 2000). Also, we use a surface albedo of 0.2 and 0.23 for MSL and InSight sites, respectively (Christensen et al., 2001; Pleskot and Miner, 1981).

First, we retrieve the surface temperatures using observation data at both MSL and InSight sites. Next, we utilize the incident solar energy flux from the Mars Climate Database v5.3 (MCD) for this study's duration. The MCD outputs are available freely and are derived using the Laboratoire de Météorologie Dynamique Mars GCM (hereafter MGCM) (Forget et al., 1998, 1999; Lefèvre et al., 2004; Madeleine et al., 2011; Navarro et al., 2014; Pottier et al., 2017; Singh, 2020; Singh et al., 2018). The MGCM is a 3-D General Circulation Model that simulates the physical and chemical state of the Martian atmosphere. The model incorporates various physical and chemical schemes such as the $\mathrm{CO}_{2}$ (Forget et al., 1998, 1999), water (Navarro et al., 2014), dust cycles (Madeleine et al., 2011; Wolff et al., 2006, 2009, 2010), and a photochemical module (Lefèvre et al., 2004).

To estimate the surface thermal inertia using Equation (7), we directly use the MCD outputs (without solving any radiative transfer), surface temperatures from the observation data, and previously known surface albedo. Next, we utilize the KRC model to determine the diurnal temperature variation for each Martian sol in our study using previously known thermal inertia values and the newly estimated thermal inertia values in this study. Finally, we compare observed diurnal temperature variation with previously known and new thermal inertia diurnal temperature profile estimates. A graphical representation of the basic algorithm used in our analysis is shown in Figure 2.

\section{Results and Discussion}

The determination of surface thermal inertia (using Equation (7)) primarily depends on three parameters: incident solar energy flux, surface albedo, and diurnal temperature variation. The observed diurnal temperature variation (for selected $L s$ ) for the MSL rover site and InSight lander site are shown in Figure 3. As expected, the local temperature changes with the position of the Sun in the sky. The temperature peaks around noon or early-afternoon hours (LTST 12-15) and has the lowest value during the early-morning hours (LTST 6-8). During the late afternoon (LTST 14-16) hours, we observe a few missing data points in the observed surface temperatures for FOV1 at the InSight mission site because of more frequent passes of the lander shadow (as FOV1 is closer to the lander as compared to FOV2). However, sufficient peak and lowest temperature values were available to compute the diurnal amplitude $(\Delta T)$ of the surface temperature. Table 1 lists the diurnal amplitude $(\Delta T)$ of the surface temperature for selected sols (Figure 3). Generally, $\Delta T$ is smaller for dust season (MY34) as compared to the non-dusty season (MY35). The significance of this will be discussed in Subsection 4.2.

\subsection{Dust Opacity and Surface Flux}

Figure 4 depicts the column dust optical depth (CDOD) for both mission sites for MY34 using the Mars Climate Sounder (MCS) observations onboard Mars Reconnaissance Orbiter (Montabone et al., 2015, 2020). Due to the LDS (Guzewich et al., 2019; Montabone et al., 2020; Streeter et al., 2020; Viúdez-Moreiras et al., 2020), showing that the dust activity during late MY34 (from $\sim \mathrm{Ls}=320^{\circ}$ to $L s=340^{\circ}$ ) is much higher. The amount of dust in the atmosphere impacts the energy flux reaching the surface. Therefore, the maximum (or minimum) surface temperatures for MY34 are higher than MY35 by about 11.0 (12.7) K, 5.4 (3.4) K, and 10.5 (3.4) $\mathrm{K}$ for MSL, InSight (FOV1), and InSight (FOV2), respectively. The in- 


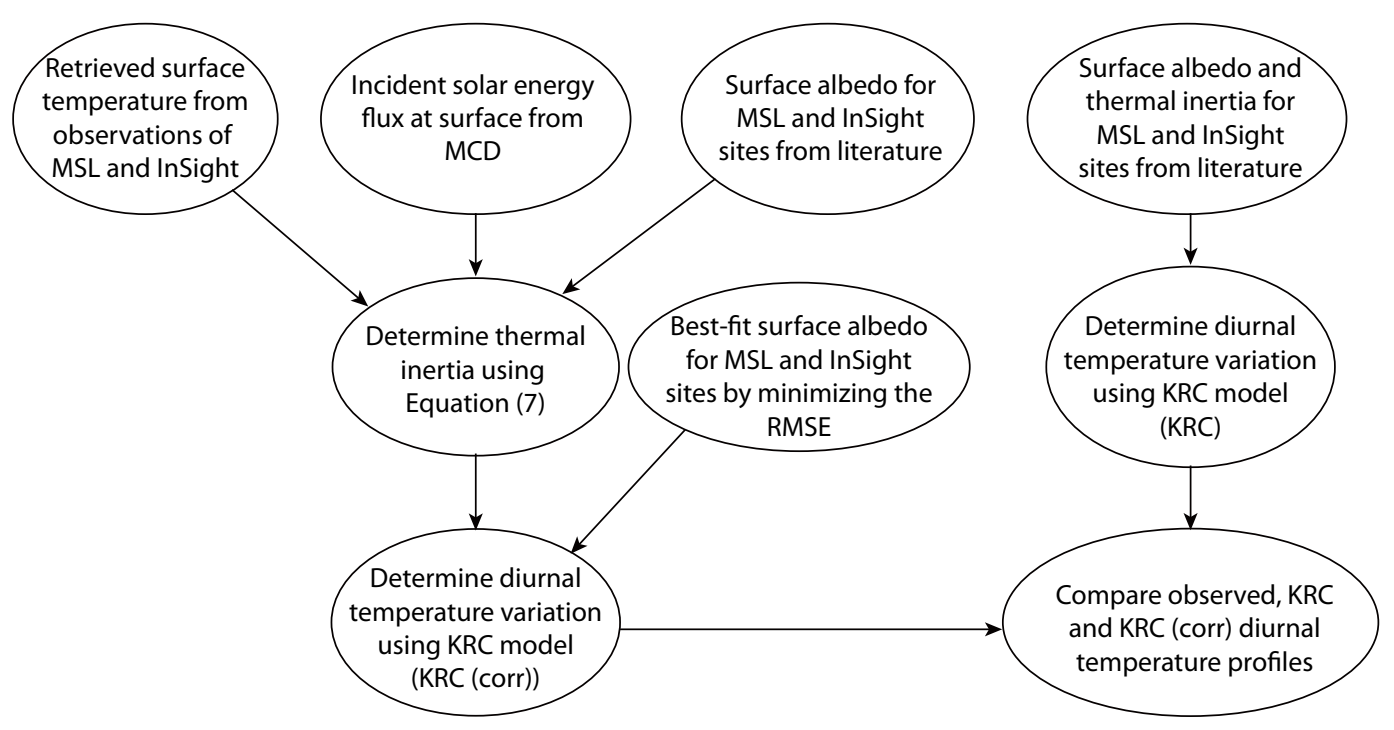

Figure 2. Flowchart of the methodology.
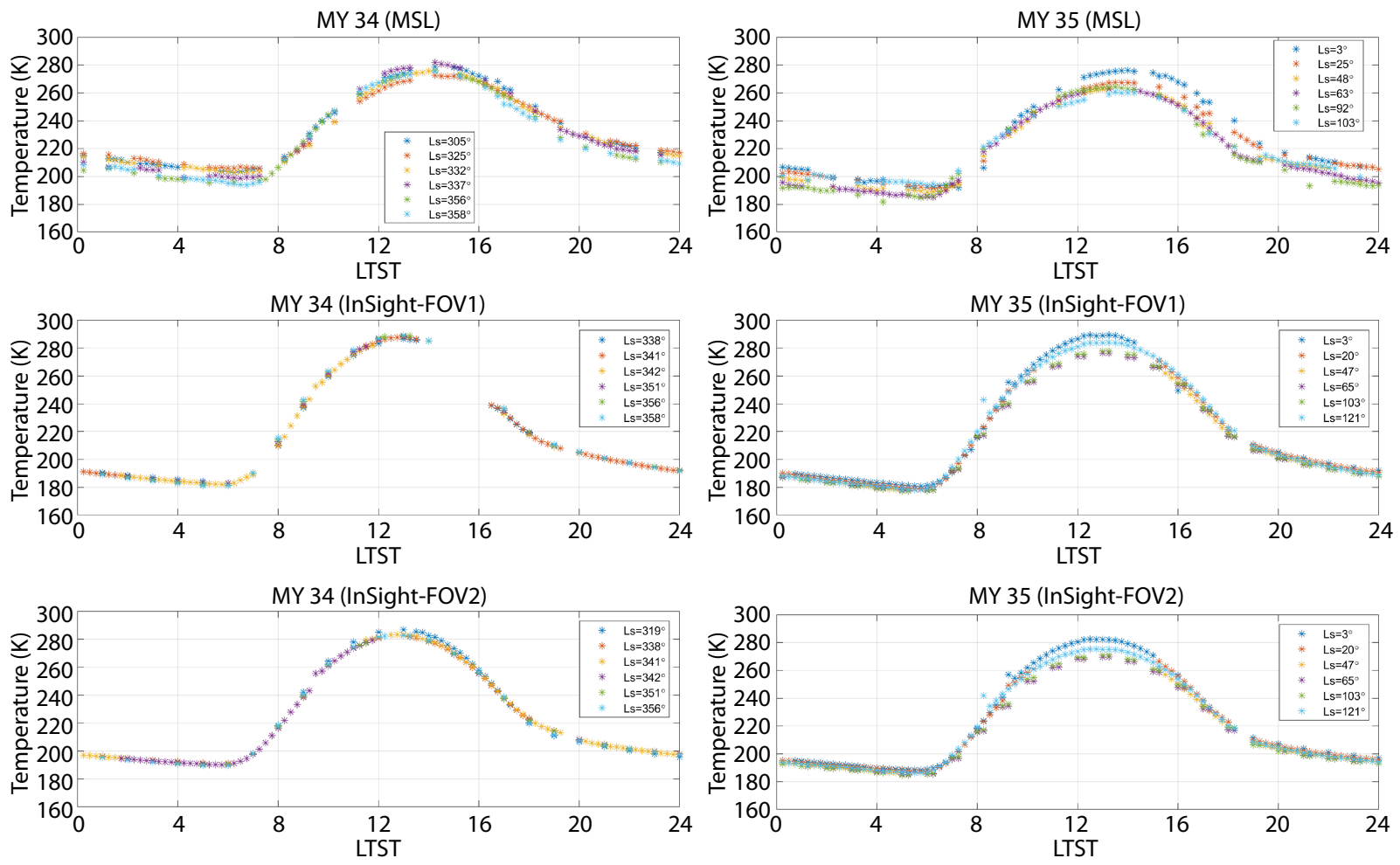

Figure 3. Variation of the surface temperature (for selected Ls) with Local True Solar Time (LTST). The left panel shows the surface temperature variation for MY34, and the right panel shows the surface temperature variation for MY35. Top row plots indicate the surface temperatures of the MSL rover site, while middle and bottom row plots indicate the surface temperatures of FOV1 and FOV2 for the InSight lander site.

cident solar energy flux at the surface is significantly impacted by the dust opacity of the atmosphere, thus any changes would affect surface temperature variation.

\subsection{Thermal Inertia Calculations}

Figure 5 depicts the estimated thermal inertia using Equation (5) for the MSL and InSight FOV1/2, showing a rise in the surface thermal inertia for all sites during high dust activity compared to the non-dusty season. This is expected as the $\Delta T$ shows the in- verse trend for the same (Table 1). The average thermal inertia in MY34 is about 444,162 , and $184 \mathrm{~J} \cdot \mathrm{m}^{-2} \cdot \mathrm{s}^{-1 / 2} \cdot \mathrm{K}^{-1}$ for the MSL, InSight (FOV1), and InSight (FOV2), respectively versus MY35 is about 319,157 , and $178 \mathrm{~J} \cdot \mathrm{m}^{-2} \cdot \mathrm{s}^{-1 / 2} \cdot \mathrm{K}^{-1}$ for the same locations. When the dust storm lifts dust from the surface, the underlying surface with high thermal inertia gets exposed. This indicates that the loose dust has much lower thermal inertia compared to underlying rocks. Therefore, dust plays a significant role in determining a planet's surface thermal response to solar flux. 
Table 1. Diurnal amplitude $(\Delta T)$ of the surface temperature $(K)$ for selected sols (Figure 3 ).

\begin{tabular}{ccccc}
\hline \multicolumn{5}{c}{ MY34 } \\
\hline Ls $\left(^{\circ}\right)$ & MSL & Ls $\left(^{\circ}\right)$ & InSight (FOV1) & InSight (FOV2) \\
\hline 305 & 114.24 & 338 & 102.87 & 92.03 \\
325 & 99.55 & 341 & 105.99 & 93.24 \\
332 & 113.77 & 342 & 100.71 & 90.09 \\
337 & 146.86 & 351 & 106.54 & 93.38 \\
356 & 151.84 & 356 & 108.04 & 93.66 \\
358 & 143.93 & 358 & 108.94 & 94.39 \\
\hline & & & MY35 & \\
\hline Ls $\left(^{\circ}\right)$ & MSL & Ls $\left(^{\circ}\right)$ & InSight (FOV1) & InSight (FOV2) \\
3 & 156.14 & 3 & 109.37 & 94.65 \\
25 & 171.08 & 20 & 108.32 & 91.94 \\
48 & 203.57 & 47 & 118.23 & 106.21 \\
63 & 171.38 & 65 & 108.23 & 91.82 \\
92 & 185.35 & 103 & 110.63 & 95.71 \\
103 & 163.55 & 121 & 109.58 & 92.19 \\
\hline
\end{tabular}

The estimated thermal inertia values at the MSL site are consistent with previous measurements (Hamilton et al., 2014; Martínez et al., 2014; Pelkey and Jakosky, 2002). However, at the InSight site, the estimated thermal inertia values during the non-dusty period are much lower than the previously known thermal inertia values (Christensen and Moore, 1992; Jakosky et al., 2000; Mellon et al., 2000). The previously known thermal inertia are estimated using orbiter data, which generally have much coarser resolution compared to the point measurements made by rovers and

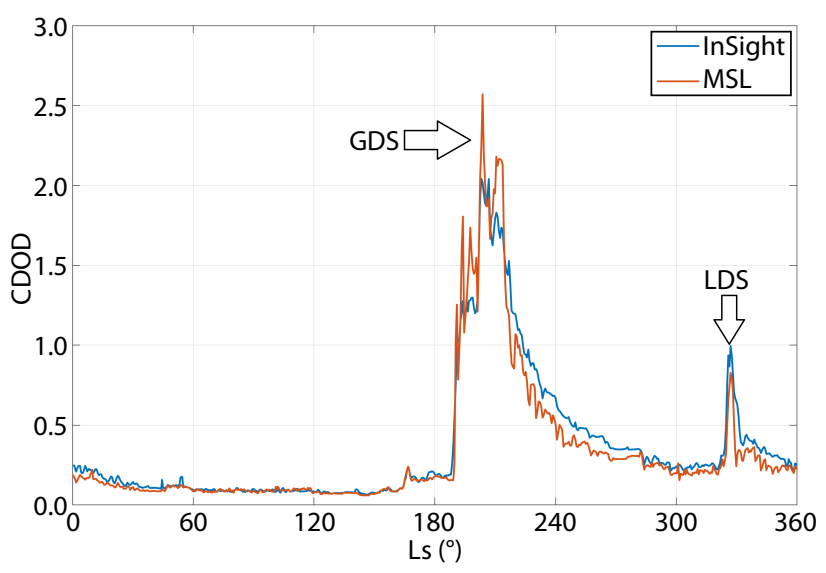

Figure 4. Column dust optical depth (CDOD) for each landing site for MY34 using the MCS observations. GDS and LDS represent Global Dust Storm and Large Dust Storm for MY34, respectively.

landers. Also, rovers and landers can track local seasonal and dynamic changes more efficiently throughout the year. However, the orbiters only get a glance at a particular site when passing directly over the site in orbit. Interestingly, the thermal inertia of FOV2 is generally higher than FOV1 for the InSight landing siteon average, the thermal inertia of FOV2 is higher by about 20 $\mathrm{J} \cdot \mathrm{m}^{-2} \cdot \mathrm{s}^{-1 / 2} \cdot \mathrm{K}^{-1}$ for the entire duration. This could be due to the FOV2 sensor capturing more surface area and looking further away from the lander than the FOV1 sensor.

\subsection{Temperature Profile Comparison with the KRC Model}

Figures 6-8 show the comparison of observed diurnal temperature profiles with the KRC-computed diurnal temperature profiles for the MSL, InSight FOV1, and InSight FOV2 regions, respectively, using previously known thermal inertia and surface albedo values

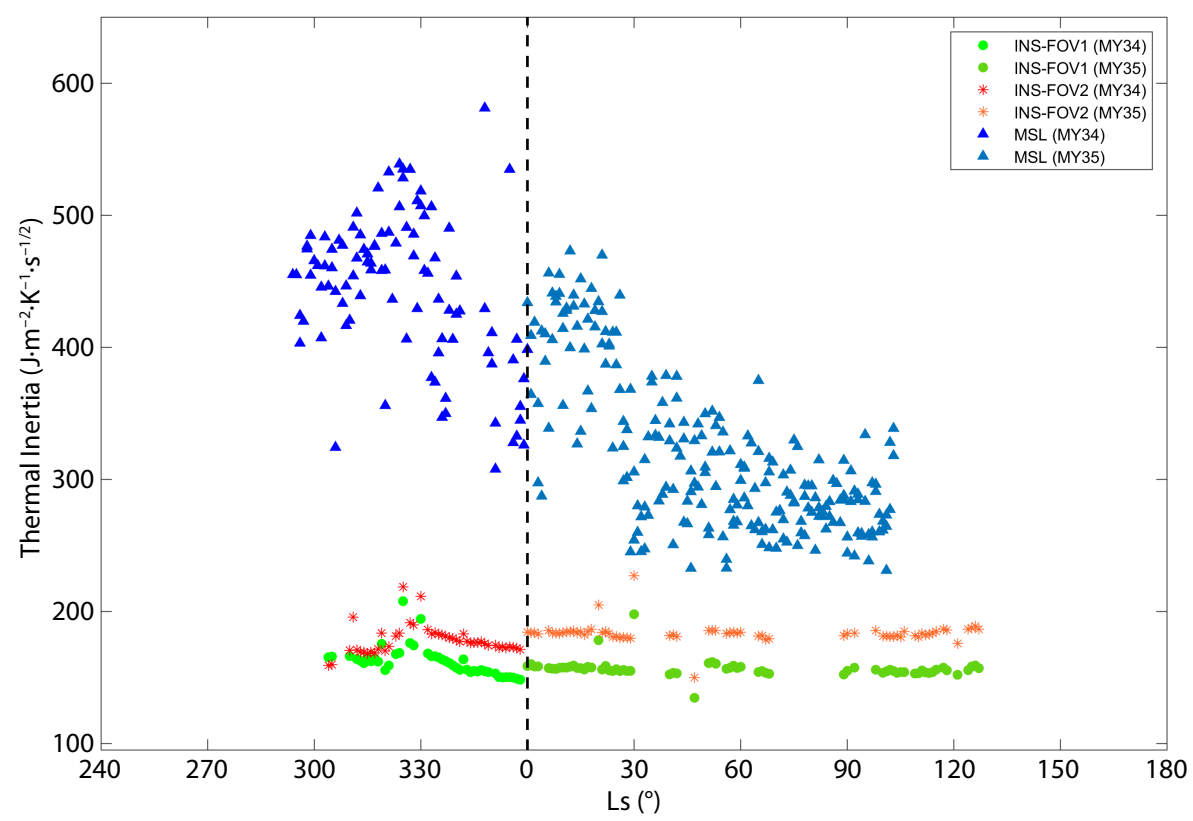

Figure 5. Estimated thermal inertia using Equation (7) and observed surface temperatures. The black dotted line indicates the boundary between MY34 and MY35. 

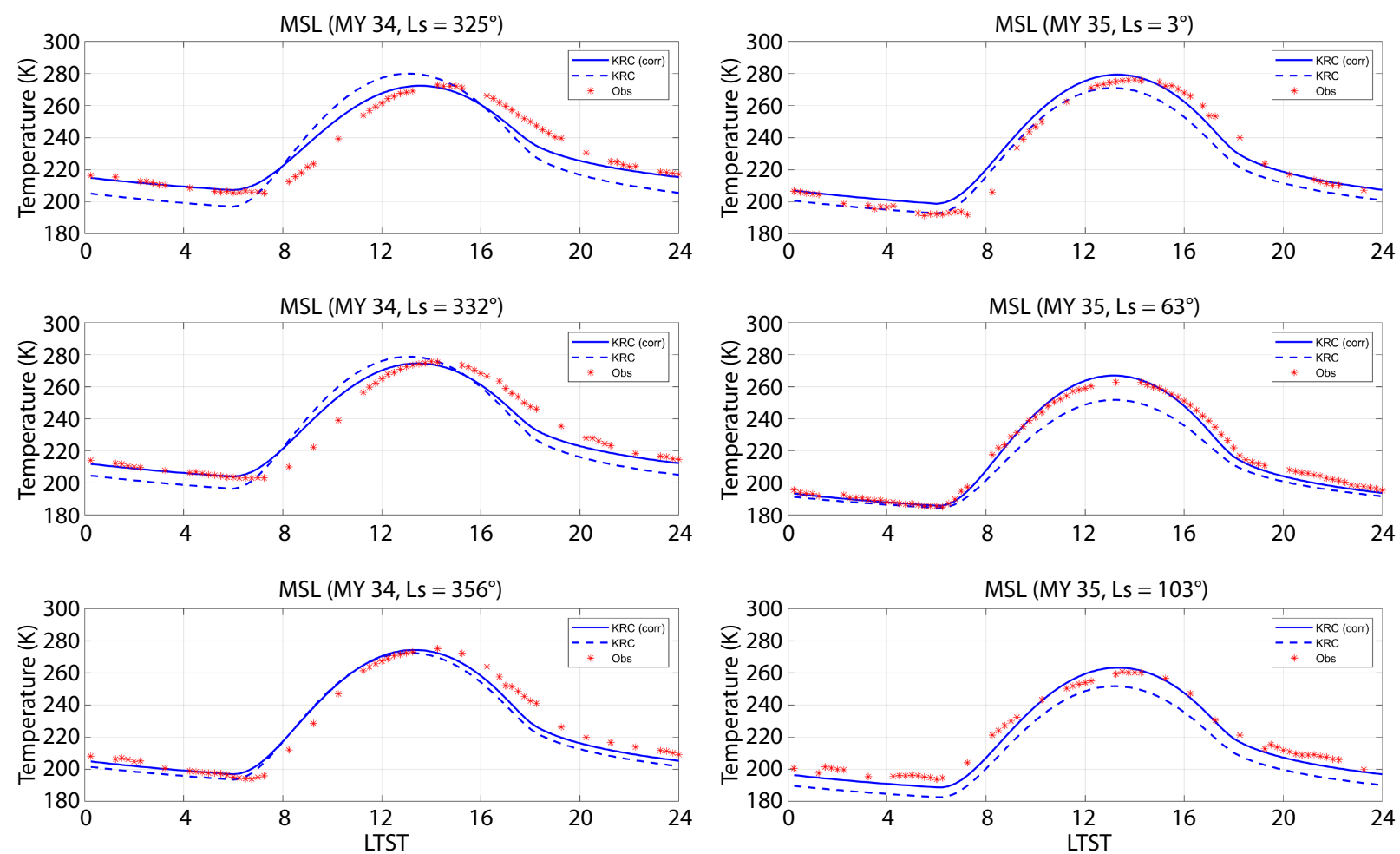

Figure 6. Comparison of observed diurnal temperature profiles at the MSL site with computed temperature profiles from the KRC model. The dotted-line profiles (KRC) are computed using previously known thermal inertia values. The solid-line profiles (KRC (corr)) are computed using new estimated thermal inertia values in this study.
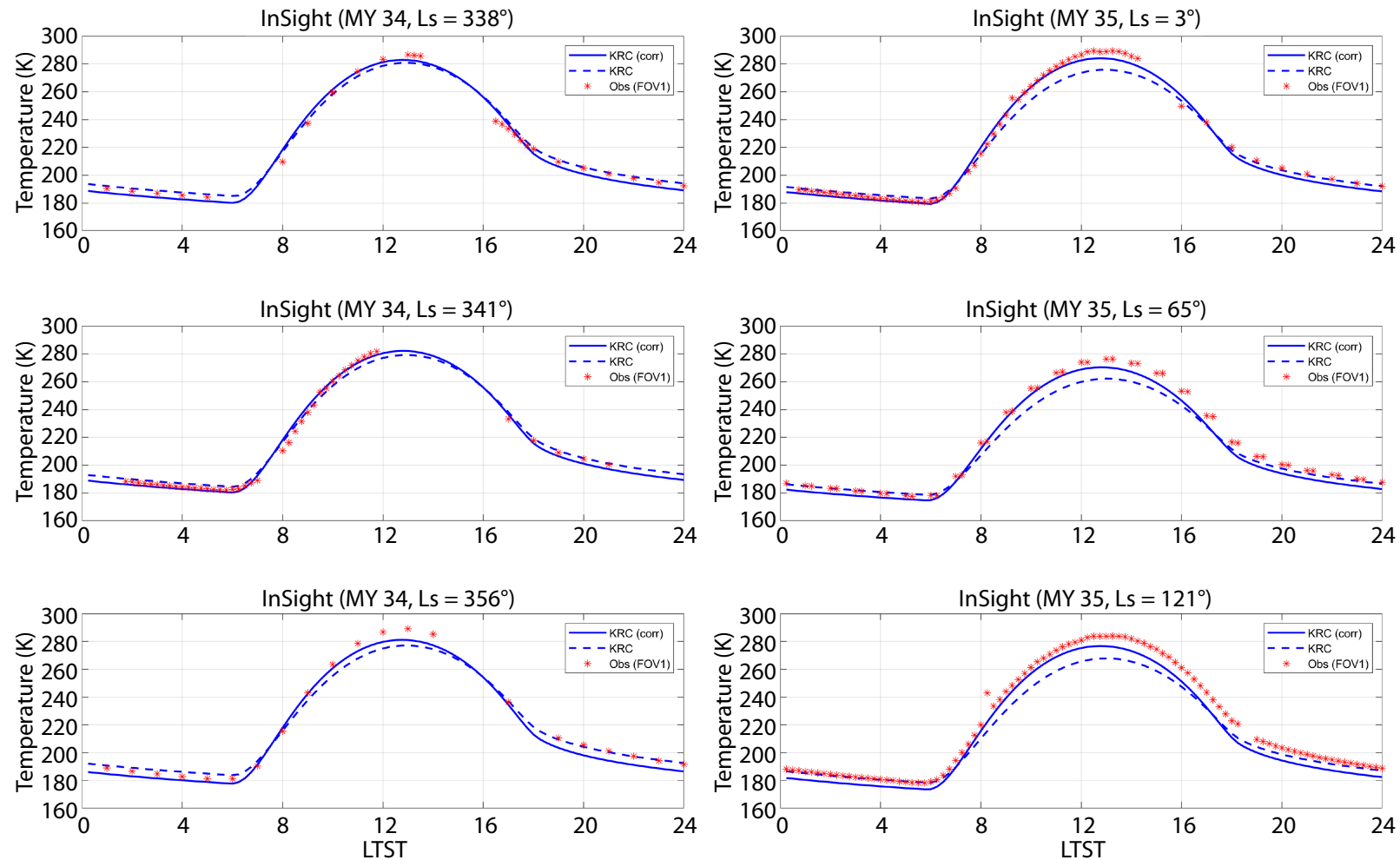

Figure 7. Same as Figure 6, but for InSight FOV1. 

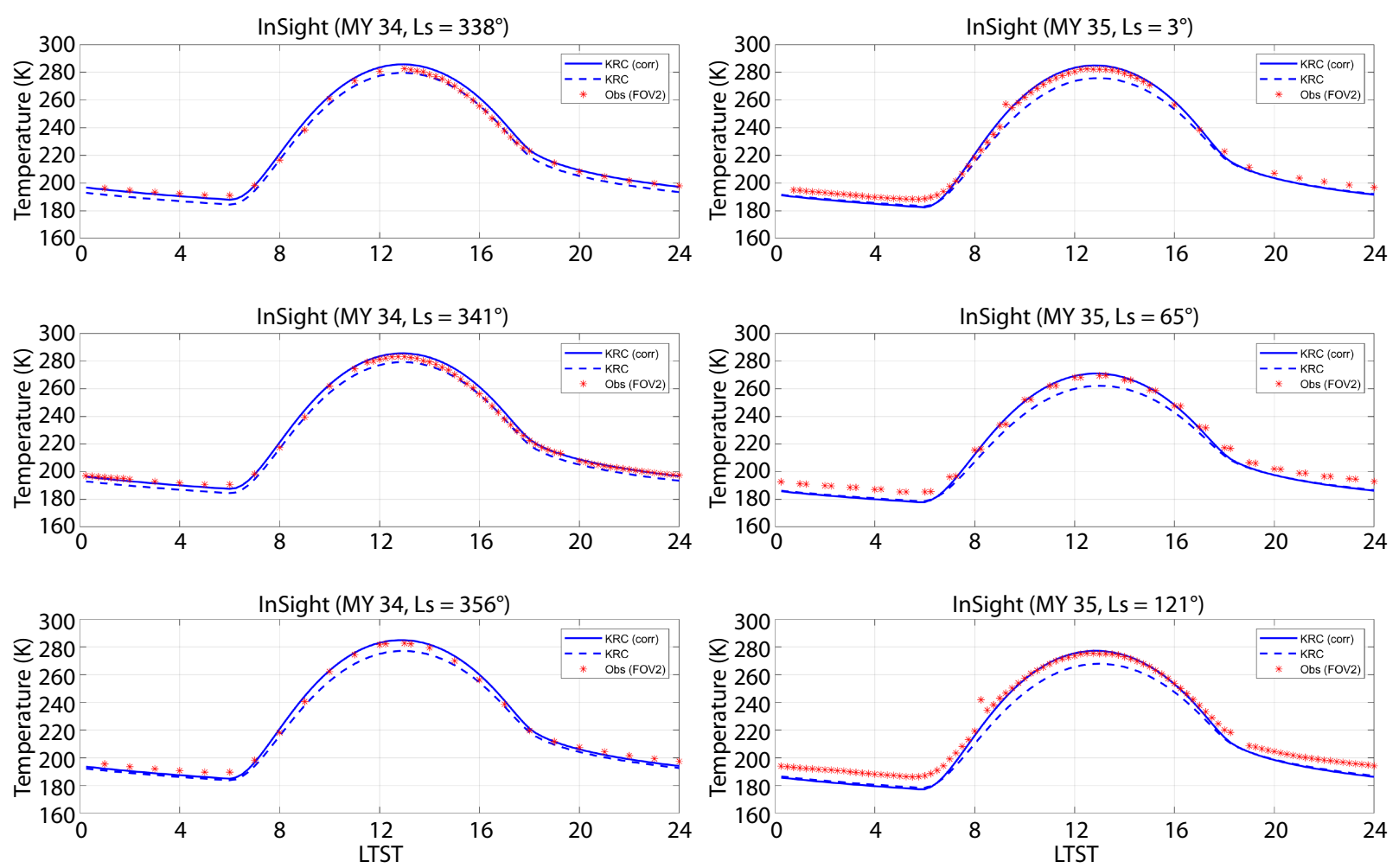

Figure 8. Same as Figures 6-7, but for InSight FOV2.

Table 2. Best-fit surface albedo for the KRC (corr) temperature profiles.

\begin{tabular}{ccc}
\hline & MY34 & MY35 \\
\hline MSL & 0.18 & 0.10 \\
InSight (FOV1) & 0.23 & 0.18 \\
InSight (FOV2) & 0.18 & 0.15 \\
\hline
\end{tabular}

(Section 3). The KRC (corr) profiles use estimated thermal inertia computed in this study and surface albedo measurements which best fit observations. The Root Mean Squared Error (RMSE) is minimized between the observed data and the model output to determine the best-fit surface albedo for the KRC (corr) temperature profiles, listed in Table 2. The surface albedo also increases during the LDS, following a similar trend as the surface thermal inertia, which indicates that the underlying surface is also more reflective than the loose dust cover on the top. The best-fit surface albedo of FOV1 is higher than that of FOV2 for all scenarios. Differences in surface albedo and thermal inertia for such nearby areas indicate significant variation in the bulk surface composition in the region, even for small distances.

Table 3 compares the RMSE temperature profile values between the default scenario and the best-fit scenario, where the former utilizes the previously known thermal inertia and surface albedo values. The MSL site observes a significant reduction in RMSE values in the best-fit scenario compared to the InSight sites. The improvement is not as considerable at InSight sites as the MSL site because of a significant amount of missing data. In MY34, the RMSE drops by about 32\%, 19\%, and $17 \%$ for the MSL, InSight (FOV1), and InSight (FOV2), respectively, whereas in MY35, the
Table 3. Comparison of RMSE values between the default scenario and the best-fit scenario. All values are in Kelvin.

\begin{tabular}{ccccc}
\hline & \multicolumn{2}{c}{ Default Scenario } & \multicolumn{2}{c}{ Best-fit Scenario } \\
\hline & MY34 & MY35 & MY34 & MY35 \\
MSL & 10.34 & 8.89 & 7.02 & 4.44 \\
InSight (FOV1) & 4.75 & 7.2 & 4.26 & 5.54 \\
InSight (FOV2) & 3.76 & 6.4 & 3.11 & 4.93 \\
\hline
\end{tabular}

RMSE drops by about $50 \%, 23 \%$, and $23 \%$ for these respective sites.

\subsection{Error Analysis}

The error in the thermal inertia determination is caused mainly by the uncertainties in the surface albedo, incident solar energy flux, and surface temperature retrievals. We estimate an overall error of about $5 \%-7 \%$ in the thermal inertia calculations, while the uncertainty in dust retrievals primarily causes the error in the incident solar flux determination. The retrieved dust CDOD has an uncertainty of $\sim 5 \%$ (Montabone et al., 2020), leading to the same uncertainty in the incident thermal flux estimation. The surface albedo retrievals have an error ranging from about 2\%-5\% (Christensen et al., 2001; Martínez et al., 2014; Pleskot and Miner, 1981). The surface temperature retrievals have an uncertainty of about $1.3 \%$ at the MSL site and $2.67 \%$ at the InSight site (Gómez-Elvira et al., 2012; Kopp et al., 2016; Spohn et al., 2018). However, the error caused due to temperature retrievals is almost negligible because Equation (7) utilizes the temperature change (instead of exact temperature values) in thermal inertia determination for a particular sol. 


\section{Conclusions}

The thermal inertia of a material depends on the material's bulk properties, namely, thermal conductivity, density, and specific heat capacity. In this study, we have estimated the surface thermal inertia of the Martian surface at the MSL rover site and the InSight lander's two different FOVs. Both mission sites observe a significant drop in the incident solar flux during the MY34 LDS. We utilize a simple derivation (Equation (7)) incorporating surface albedo, incident solar energy flux, and diurnal temperature change for the surface thermal inertia determination. The current approach provides thermal inertia values with reasonable accuracy, all without knowing the entire radiative transfer solution through the Martian atmosphere nor solving complicated numerical models. The drawback of this approach is it relies heavily on daily temperature measurements (maximum and minimum) and surface flux estimation. In some scenarios, when crucial temperature data is either missing (due to instrumental shutdown) or unreliable due to a malfunction, then the thermal inertia estimates would be erroneous by the same order.

We have compared the thermal inertia during dusty (MY34) and non-dusty (MY35) periods of Mars, which includes the LDS during MY34. The thermal inertia of all three sites shows a significant increase during the dusty period, indicating exposure of underlying rocks during the LDS. The average thermal inertia in MY34 is about 39.2\%, 3.7\%, and 3.4\% higher than MY35 average thermal inertia for the MSL, InSight FOV1, and FOV2, respectively. The relatively significant change at the MSL site could probably be caused due to topographical effects at the respective location. The estimated thermal inertia values during the non-dusty season at the MSL site are consistent with previous studies. However, thermal inertia values at the InSight sites are much lower than previously known values, and the average thermal inertia (nondusty season) at the FOV1 region is consistently lower by about $12 \%$ than the FOV2 region.

In this study, we compare the diurnal surface temperature variation using the KRC model with previously known and estimated thermal inertia. We also perform a best-fit analysis between the observed and the estimated temperature variation by varying the surface albedo for both sites. The surface albedo also increases during the LDS, indicating that the underlying surface is both more thermally resistant and more reflective than the overlying loose dust. The best-fit surface albedo of FOV1 is about $28 \%$ and $20 \%$ higher than FOV1 surface albedo for MY34 and MY35, respectively. Although the FOV1 and FOV2 regions are relatively close to each other, surface albedo and thermal inertia differences indicate significant variation in the region's bulk surface composition, even for small distances. The surface bulk properties play a significant role in a planet's energy budget. The improvement in RMSE is not as considerable at the InSight sites as the MSL site because of a significant amount of missing data. In the future, the surface thermal inertia and surface albedo could be better constrained with more data availability.

\section{Data Availability}

The REMS and HP3/RAD data are publicly available at https://pdsatmospheres.nmsu.edu/data_and_services/atmospheres_data/M
ARS/curiosity/rems.html and https://doi.org/10.17189/1517568, respectively. The web version of Mars Climate Database v5.3 created using the MGCM can be interactively accessed on the web at: http://www-mars.Imd.jussieu.fr/mars/access.html. The output/derived results data in this work are available at https://github.com/ sdeepak88/thermal_inertia_2021.

Authors' contributions: DS performed the conceptualization and methodology. DS and SU performed analysis and discussions. DS and SU derived InSight and REMS data respectively. All authors read and approved the final manuscript. Funding: This work was partially supported by the DST-INSPIRE Faculty Award. Competing Interests: Authors declare that they have no competing interests.

\section{References}

Banerdt, W. B., Smrekar, S. E., Banfield, D., Giardini, D., Golombek, M., Johnson, C. L., Lognonné, P., Spiga, A., Spohn, T., ... Wieczorek, M. (2020). Initial results from the InSight mission on Mars. Nat. Geosci., 13(3), 183-189. https://doi.org/10.1038/s41561-020-0544-y

Banfield, D., Spiga, A., Newman, C., Forget, F., Lemmon, M., Lorenz, R., Murdoch, N., Viudez-Moreiras, D., Pla-Garcia, J., ... Banerdt, W. B. (2020). The atmosphere of Mars as observed by InSight. Nat. Geosci., 13(3), 190-198. https://doi.org/10.1038/s41561-020-0534-0

Christensen, P., and Moore, H. J. (1992). The Martian surface layer. In H. H. Kieffer (Eds.), Mars, 44, 686-729. Tucson: University of Arizona Press, Space Science Series.

Christensen, P. R., Bandfield, J. L., Hamilton, V. E., Ruff, S. W., Kieffer, H. H., Titus, T. N., Malin, M. C., Morris, R. V., Lane, M. D., ... Greenfield, M. (2001). Mars Global Surveyor Thermal Emission Spectrometer experiment: Investigation description and surface science results. J. Geophys. Res.: Planets, 106(E10), 23823-23871. https://doi.org/10.1029/2000JE001370

Forget, F., Hourdin, F., and Talagrand, O. (1998). $\mathrm{CO}_{2}$ snowfall on Mars: Simulation with a general circulation model. Icarus, 131(2), 302-316. https://doi.org/10.1006/icar.1997.5874

Forget, F., Hourdin, F., Fournier, R., Hourdin, C., Talagrand, O., Collins, M., Lewis, S. R., Read, P. L., and Huot, J.-P. (1999). Improved general circulation models of the Martian atmosphere from the surface to above $80 \mathrm{~km}$. J. Geophys. Res.: Planets, 104(E10), 24155-24175. https://doi.org/10.1029/1999JE001025

Golombek, M., Warner, N. H., Grant, J. A., Hauber, E., Ansan, V., Weitz, C. M., Williams, N., Charalambous, C., Wilson, S. A., ... Banerdt, W. B. . (2020). Geology of the InSight landing site on Mars. Nat. Commun., 11(1), 1014. https://doi.org/10.1038/s41467-020-14679-1

Gómez-Elvira, J. , Armiens, C. , Castañer, L. , Domínguez, M. , Genzer, M. , Gómez, F. , Haberle, R. , Harri, A. -M. , Jiménez, V. , ... Martín-Torres, J. (2012). REMS: The environmental sensor suite for the mars science laboratory rover. Space Sci. Rev., 170(1), 583-640. https://doi.org/10.1007/s11214-012-9921-1

Gómez-Elvira, J. (2013a). Mars Science Laboratory Rover Environmental Monitoring Station RDR Data V1.0, MSL-M-REMS-4-ENVEDR-V1.0. NASA Planet. Data Syst.

Gómez-Elvira, J. (2013b). Mars Science Laboratory Rover Environmental Monitoring Station RDR Data V1.0, MSL-M-REMS-5-MODRDR-V1.0. NASA Planet. Data Syst.

Guzewich, S. D., Lemmon, M., Smith, C. L., Martínez, G., de Vicente-Retortillo, Á., Newman, C. E., Baker, M., Campbell, C., Cooper, B., ... Mier, M.-P. Z. (2019). Mars science laboratory observations of the 2018/Mars Year 34 global dust storm. Geophys. Res. Lett., 46(1), 71-79. https://doi.org/10.1029/2018GL080839

Haberle, R. M., Gómez-Elvira, J., de la Torre Juárez, M., Harri, A.-M., Hollingsworth, J. L., Kahanpää, H., Kahre, M. A., Lemmon, M., Martín-Torres, 
F. J., ... Zorzano-Mier, M.-P. (2014). Preliminary interpretation of the REMS pressure data from the first 100 sols of the MSL mission. J. Geophys. Res.: Planets, 119(3), 440-453. https://doi.org/10.1002/2013JE004488

Hamilton, V. E., Vasavada, A. R., Sebastián, E., de la Torre Juárez, M., Ramos, M., Armiens, C., Arvidson, R. E., Carrasco, I., Christensen, P. R., ... Zorzano, M.-P. (2014). Observations and preliminary science results from the first 100 sols of MSL Rover Environmental Monitoring Station ground temperature sensor measurements at Gale Crater. J. Geophys. Res.: Planets, 119(4), 745-770. https://doi.org/10.1002/2013JE004520

Hamm, M., Grott, M., Kührt, E., Pelivan, I., and Knollenberg, J. (2018). A method to derive surface thermophysical properties of asteroid (162173) Ryugu (1999JU3) from in-situ surface brightness temperature measurements. Planet. Space Sci., 159, 1-10. https://doi.org/10.1016/j.pss.2018.03.017

Jakosky, B. M. (1986). On the thermal properties of Martian fines. Icarus, 66(1), 117-124. https://doi.org/10.1016/0019-1035(86)90011-4

Jakosky, B. M., Mellon, M. T., Kieffer, H. H., Christensen, P. R., Varnes, E. S., and Lee, S. W. (2000). The thermal inertia of Mars from the Mars Global Surveyor Thermal Emission Spectrometer. J. Geophys. Res.: Planets, 105(E4), 9643-9652. https://doi.org/10.1029/1999JE001088

Kieffer, H. H. (2013). Thermal model for analysis of Mars infrared mapping. J. Geophys. Res.: Planets, 118(3), 451-470. https://doi.org/10.1029/2012JE004164

Kopp, E., Mueller, N., Grott, M., Walter, I., Knollenberg, J., Hanschke, F., Kessler, E., and Meyer, H.-G. (2016). HP3-RAD: A compact radiometer design with on-site calibration for in-situ exploration. In. Proceedings of the SPIE 9973, Infrared Remote Sensing and Instrumentation XXIV, 9973, 99730T. San Diego: SPIE. https://doi.org/10.1117/12.2236190

Lefèvre, F., Lebonnois, S., Montmessin, F., and Forget, F. (2004). Threedimensional modeling of ozone on Mars. J. Geophys. Res.: Planets, 109(E7). https://doi.org/10.1029/2004JE002268

Madeleine, J.-B., Forget, F., Millour, E., Montabone, L., and Wolff, M. J. (2011). Revisiting the radiative impact of dust on Mars using the LMD Global Climate Model. J. Geophys. Res.: Planets, 116(E11). https://doi.org/10.1029/2011JE003855

Martínez, G. M., Rennó, N., Fischer, E., Borlina, C. S., Hallet, B., de la Torre Juárez, M., Vasavada, A. R., Ramos, M., Hamilton, V., ... Haberle, R. M. . (2014). Surface energy budget and thermal inertia at Gale Crater: Calculations from ground-based measurements. J. Geophys. Res.: Planets, 119(8), 1822-1838. https://doi.org/10.1002/2014JE004618

Mellon, M. T., Jakosky, B. M., Kieffer, H. H., and Christensen, P. R. (2000). Highresolution thermal inertia mapping from the mars global surveyor thermal emission spectrometer. Icarus, 148(2), 437-455. https://doi.org/10.1006/icar.2000.6503

Montabone, L., Forget, F., Millour, E., Wilson, R. J., Lewis, S. R., Cantor, B., Kass, D., Kleinböhl, A., Lemmon, M. T., ... Wolff, M. J. (2015). Eight-year climatology of dust optical depth on Mars. Icarus, 251, 65-95. https://doi.org/10.1016/j.icarus.2014.12.034

Montabone, L., Spiga, A., Kass, D. M., Kleinböhl, A., Forget, F., and Millour, E. (2020). Martian Year 34 column dust climatology from mars climate sounder observations: Reconstructed maps and model simulations. J. Geophys. Res.: Planets, 125(8), e2019JE006111. https://doi.org/10.1029/2019JE006111

Morgan, P., Grott, M., Knapmeyer-Endrun, B., Golombek, M., Delage, P., Lognonné, P., Piqueux, S., Daubar, I., Murdoch, N., ... Kedar, S. (2018). A PreLanding Assessment of Regolith Properties at the InSight Landing Site. Space Sci. Rev., 214(6), 104. https://doi.org/10.1007/s11214-018-0537-y

Mueller, N. T., Knollenberg, J., Grott, M., Kopp, E., Walter, I., Krause, C., Hudson, T., Spohn, T., and Smrekar, S. (2020). Calibration of the $\mathrm{HP}^{3}$ radiometer on InSight. Earth Space Sci., 7(5), e2020EA001086. https://doi.org/10.1029/2020EA001086

Navarro, T., Madeleine, J.-B., Forget, F., Spiga, A., Millour, E., Montmessin, F., and
Määttänen, A. (2014). Global climate modeling of the Martian water cycle with improved microphysics and radiatively active water ice clouds. J. Geophys. Res.: Planets, 119(7), 1479-1495. https://doi.org/10.1002/2013JE004550

Parro, L. M., Jiménez-Díaz, A., Mansilla, F., and Ruiz, J. (2017). Present-day heat flow model of Mars. Sci. Rep., 7(1), 45629. https://doi.org/10.1038/srep45629

Pelkey, S. M., and Jakosky, B. M. (2002). Surficial geologic surveys of gale crater and melas chasma, mars: integration of remote-sensing data. Icarus, 160(2), 228-257. https://doi.org/10.1006/icar.2002.6978

Piqueux, S., Kopp, E., Spohn, T., Smrekar, S. E., Knollenberg, J., Hudson, T. L., Krause, C., Plesa, A. C., Siegler, M., and Spiga, A. (2019). HP3 radiometer measurements from the Mars mission insight.

Pleskot, L. K., and Miner, E. D. (1981). Time variability of Martian bolometric albedo. Icarus, 45(1), 179-201. https://doi.org/10.1016/0019-1035(81) 90013-0

Pottier, A., Forget, F., Montmessin, F., Navarro, T., Spiga, A., Millour, E., Szantai, A., and Madeleine, J.-B. (2017). Unraveling the martian water cycle with high-resolution global climate simulations. Icarus, 291, 82-106. https://doi.org/10.1016/j.icarus.2017.02.016

Putzig, N. E., Mellon, M. T., Kretke, K. A., and Arvidson, R. E. (2005). Global thermal inertia and surface properties of Mars from the MGS mapping mission. Icarus, 173(2), 325-341. https://doi.org/10.1016/j.icarus.2004.08.017

Putzig, N. E., and Mellon, M. T. (2007). Apparent thermal inertia and the surface heterogeneity of Mars. Icarus, 191(1), 68-94. https://doi.org/10.1016/j.icarus.2007.05.013

Savijärvi, H. I., Harri, A.-M., and Kemppinen, O. (2015). Mars Science Laboratory diurnal moisture observations and column simulations. J. Geophys. Res.: Planets, 120(5), 1011-1021. https://doi.org/10.1002/2014JE004732

Singh, D., Flanner, M. G., and Millour, E. (2018). Improvement of Mars surface snow albedo modeling in LMD Mars GCM with SNICAR. J. Geophys. Res.: Planets, 123(3), 780-791. https://doi.org/10.1002/2017JE005368

Singh, D. (2020). Impact of surface Albedo on Martian photochemistry. Earth Planet. Phys., 4(3), 206-211. https://doi.org/10.26464/epp2020025

Smith, D. E., Zuber, M. T., Solomon, S. C., Phillips, R. J., Head, J. W., Garvin, J. B., Banerdt, W. B., Muhleman, D. O., Pettengill, G. H., ... Duxbury, T. C. (1999). The global topography of mars and implications for surface evolution. Science, 284(5419), 1495-1503. https://doi.org/10.1126/science.284.5419.1495

Spiga, A. , and Forget, F. (2009). A new model to simulate the Martian mesoscale and microscale atmospheric circulation: Validation and first results. J. Geophys. Res.: Planets, 114(E2). https://doi.org/10.1029/ 2008JE003242

Spohn, T., Grott, M., Smrekar, S. E., Knollenberg, J., Hudson, T. L., Krause, C., Müller, N., Jänchen, J., Börner, A., ... Banerdt, W. B. . (2018). The heat flow and physical Properties Package (HP3) for the InSight mission. Space Sci. Rev., 214(5), 96. https://doi.org/10.1007/s11214-018-0531-4

Streeter, P. M., Lewis, S. R., Patel, M. R., Holmes, J. A., and Kass, D. M. (2020). Surface warming during the 2018/Mars Year 34 global dust storm. Geophys. Res. Lett., 47(9), e2019GL083936. https://doi.org/10.1029/2019GL083936

Tian, J., Su, H. B., He, H. L., and Sun, X. M. (2015). An empirical method of estimating soil thermal inertia. Adv. Meteorol., 2015, e428525. https://doi.org/10.1155/2015/428525

Vasavada, A. R., Piqueux, S., Lewis, K. W., Lemmon, M. T., and Smith, M. D. (2017). Thermophysical properties along Curiosity's traverse in Gale crater, Mars, derived from the REMS ground temperature sensor. Icarus, 284, 372-386. https://doi.org/10.1016/j.icarus.2016.11.035

Viúdez-Moreiras, D., Newman, C. E., Forget, F., Lemmon, M., Banfield, D., Spiga, A., Lepinette, A., Rodriguez-Manfredi, J. A., Gómez-Elvira, J., ... Grott, M. (2020). Effects of a large dust storm in the near-surface atmosphere as 
measured by insight in elysium planitia, mars. Comparison with contemporaneous measurements by Mars science laboratory. J. Geophys. Res.: Planets,, 125(9), e2020JE006493.

https://doi.org/10.1029/2020JE006493

Vu, T. H., Piqueux, S., Choukroun, M., Edwards, C. S., Christensen, P. R., and Glotch, T. D. (2019). Low-temperature specific heat capacity measurements and application to Mars thermal modeling. Icarus, 321, 824-840.

https://doi.org/10.1016/j.icarus.2018.10.004

Wall, M. 2012. Touchdown! Huge NASA rover lands on Mars. Space. Com. https://www.space.com/16932-mars-rover-curiosity-landing-success.html

Wang, J., Bras, R.L. , Sivandran, G., and Knox, R. G. (2010). A simple method for the estimation of thermal inertia. Geophys. Res. Lett., 37(5), L05404. https://doi.org/10.1029/2009GL041851

Wolff, M. J., Smith, M. D., Clancy, R. T., Spanovich, N., Whitney, B. A., Lemmon, M. T., Bandfield, J. L., Banfield, D., Ghosh, A., ... Squyres, S. W. (2006).
Constraints on dust aerosols from the Mars exploration rovers using MGS overflights and Mini-TES. J. Geophys. Res.: Planets, 111 (E12). https://doi.org/10.1029/2006JE002786

Wolff, M. J., Smith, M. D., Clancy, R. T., Arvidson, R., Kahre, M., Seelos, F., Murchie, S., and Savijärvi, H. (2009). Wavelength dependence of dust aerosol single scattering albedo as observed by the compact reconnaissance imaging spectrometer. J. Geophys. Res.: Planets, 114(E2). https://doi.org/10.1029/2009JE003350

Wolff, M. J., Todd Clancy, R., Goguen, J. D., Malin, M. C., and Cantor, B. A. (2010). Ultraviolet dust aerosol properties as observed by MARCI. Icarus, 208(1), 143-155. https://doi.org/10.1016/j.icarus.2010.01.010

Zuber, M. T., Smith, D. E., Solomon, S. C., Muhleman, D. O., Head, J. W., Garvin, J. B., Abshire, J. B., and Bufton, J. L. (1992). The Mars observer laser altimeter investigation. J. Geophys. Res.: Planets, 97(E5), 7781-7797.

https://doi.org/10.1029/92JE00341 